\title{
Magnetized Directly-Driven ICF Capsules: Increased Instability Growth from Non-Uniform Laser Drive
}

C. A. Walsh, ${ }^{1}$ A. J. Crilly, ${ }^{2}$ and J. P. Chittenden ${ }^{2}$

${ }^{1)}$ Lawrence Livermore National Laboratory ${ }^{\text {a) }}$

${ }^{2)}$ Blackett Laboratory, Imperial College London

(Dated: 24 July 2020)

Simulations anticipate increased perturbation growth from nonuniform laser heating for magnetized directdrive implosions. At the capsule pole, where the magnetic field is normal to the ablator surface, the field remains in the conduction zone and suppresses non-radial thermal conduction; in unmagnetized implosions this non-radial heat-flow is crucial in mitigating laser heating imbalances. Single-mode simulations show the magnetic field particularly amplifying short wavelength perturbations, whose behavior is dominated by thermal conduction. The most unstable wavelength can also become shorter. 3D multi-mode simulations of the capsule pole reinforce these findings, with increased perturbation growth anticipated across a wide range of scales. The results indicate that high-gain spherical direct-drive implosions require greater constraints on the laser heating uniformity when magnetized.

\section{INTRODUCTION}

Applying a magnetic field to inertial confinement fusion (ICF) capsules is a method of reducing thermal conduction losses from the compressed hot-spot. Previous experiments at the OMEGA Laser Facility used an $8 \mathrm{~T}$ magnetic field to improve the yield and ion temperature by $30 \%$ and $15 \%$ respectively ${ }^{4}$. Continual advancements to the MIFEDS coils at the OMEGA Laser Facility means that initial field strengths of $50 \mathrm{~T}$ are now plausible ${ }^{26}$. Simulations predict larger yield enhancements for targets with a higher convergence and applied field strength ${ }^{23,30}$. The study of magnetized perturbation growth naturally becomes more important in a highconvergence system; while 3D simulations have been conducted for the capsule stagnation ${ }^{30}$, little published work exists on the effect of magnetic fields on the drive-phase of spherical implosions.

Magnetic flux compression is pivotal to the magnetized ICF concept. The formed hot-spot becomes dense, typically requiring magnetic fields over $1000 \mathrm{~T}$ to affect thermal conductivities. To reach these field strengths the magnetic flux must be compressed with the bulk plasma. Much of the research into the drive-phase of magnetized implosions has been focused on the efficacy of magnetic flux compression ${ }^{7,13}$. Theoretically (for a typical implosion on OMEGA) the implosion velocity must be greater than $6000 \mathrm{~m} / \mathrm{s}$ for the magnetic field compression to overcome the diffusion ${ }^{13}$. Magnetic flux compression has been confirmed with a laser driver experimentally; proton radiography indicated magnetic fields amplified from $5 \mathrm{~T}$ to greater than $2000 \mathrm{~T}$ in the hot-spot of a cylindrical implosion ${ }^{10,18}$. While previous research into the drivephase showed the plasma having an effect on the magnetic field, this paper suggests that the magnetic field can also change the capsule drive, with enhanced perturbation growth for directly-driven implosions.

\footnotetext{
a) Electronic mail: walsh34@llnl.gov
}

Unmagnetized acceleration-phase Rayleigh-Taylor instability growth has been well studied theoretically ${ }^{25}$, computationally $^{16,24}$ and experimentally ${ }^{1,28}$. Laser drive asymmetries can occur over long wavelengths due to target positioning errors and beam power imbalances ${ }^{16}$, while short wavelengths are driven by asymmetries within a single beam ${ }^{24}$. Two distinct processes mitigate these power imbalances in directly-driven implosions: thermal smoothing ${ }^{3}$ and ablation ${ }^{25}$. Thermal smoothing occurs between the critical density surface (where the laser energy is deposited) and the ablation front; in a nonuniformly driven target the heat-flow is not purely radial, and the energy deposition is more symmetric at the ablation front than in the laser absorption region. The drive is then subject to the ablative-Rayleigh-Taylor instability, where the classical Rayleigh-Taylor growth rate is reduced for high mode numbers. The smoothing acts to reduce the driving laser heating perturbation, while ablation makes the system intrinsically more Rayleigh-Taylor stable to all perturbation sources. While these two processes are clearly related to thermal conduction, applying a magnetic field to the acceleration phase is found in this paper to predominantly decrease thermal smoothing.

The transport of magnetic field in an extended-MHD plasma is governed by the equation ${ }^{31}$ :

$$
\frac{\partial \underline{B}}{\partial t}=-\nabla \times \frac{\alpha_{\|}}{\mu_{0} e^{2} n_{e}^{2}} \nabla \times \underline{B}+\nabla \times\left(\underline{v}_{B} \times \underline{B}\right)+\nabla \times \frac{\nabla P_{e}}{e n_{e}}
$$

where the first term is magnetic diffusion, the second is the field advection at velocity $\underline{v}_{B}$ and the final is the Biermann Battery term, which acts as a source for magnetic flux. The advection velocity consists of the bulk plasma flow, motion due to temperature gradients and motion due to electrical currents in the plasma.

Assuming magnetic fields realizable over target scales $\left(B_{0} \leq 50 T\right)$, the temperature gradient driven transport is much greater than the electrical current driven transport in direct-drive conditions. The magnetic field advection velocity $\underline{v}_{B}$ can then be simplified ${ }^{31}$ : 


$$
\underline{v}_{B}=\underline{v}-\gamma_{\perp} \nabla T_{e}-\gamma_{\wedge}\left(\underline{\hat{b}} \times \nabla T_{e}\right)
$$

When neglecting advection of magnetic flux by electrical currents, a magnetic diffusivity of $\alpha_{\perp}$ is used instead of $\alpha_{\|}$in equation 1.

The magnetic transport by temperature gradients can be readily compared with the thermal conduction in the presence of a magnetic field ${ }^{31}$ :

$$
\begin{array}{lll}
\underline{v}_{N} & = & -\gamma_{\perp} \nabla_{\perp} T_{e}-\gamma_{\wedge} \underline{\hat{b}} \times \nabla T_{e} \\
\underline{q}_{\kappa} & =-\kappa_{\|} \nabla_{\|} T_{e} & -\kappa_{\perp} \nabla_{\perp} T_{e}-\kappa_{\wedge} \underline{\hat{b}} \times \nabla T_{e}
\end{array}
$$

The $\kappa_{\|}$term represents the heat-flow along magnetic field lines, which is unaffected by plasma magnetization. There is no equivalent term in the magnetic transport, as magnetic field lines do not move parallel with themselves. The $\kappa_{\perp}$ term is the thermal conduction perpendicular to the magnetic field, which is suppressed by magnetization. The Nernst magnetic transport coefficient $\left(\gamma_{\perp}\right)$ is similar in magnitude to the perpendicular thermal conductivity $\left(\kappa_{\perp}\right)$ for all magnetizations ${ }^{11}$; wherever the thermal conduction is large, so is the transport of magnetic field by Nernst. The magnetization is routinely quantified by the Hall Parameter ${ }^{15}$ :

$$
\omega_{e} \tau_{e} \propto \frac{|\underline{B}| T_{e}^{3 / 2}}{n_{e}}
$$

i.e. low density and higher temperature plasma requires a lower magnetic field strength to become magnetized.

Figure 1 illustrates the heat-flow components relative to the magnetic field lines for a symmetrically-driven implosion. $q_{\|}=-\kappa_{\|} \nabla_{\|} T_{e}$ is the heat-flow along magnetic field lines, $q_{\perp}=-\kappa_{\perp} \nabla_{\perp} T_{e}$ is the heat-flow perpendicular to the field lines and $q_{\wedge}=-\kappa_{\wedge} \underline{\hat{b}} \times \nabla T_{e}$ is the Righi-Leduc heat-flow.

Utilizing the similarity between the thermal and magnetic transport (equations 3 and 4), the transport of magnetic field in a direct-drive ablation front becomes apparent. In the same way that the thermal energy is transported into the ablation front from the critical density surface against the bulk flow of plasma, so magnetic field is transported into the ablation surface by the Nernst term ${ }^{19}$. This is particularly true at the waist, where the radially-dominated advection velocities are perpendicular to the magnetic field. While the Nernst term prevents the magnetic flux in the shell from expanding into the corona, it also effectively transports the flux into the cold and dense ablation front, which requires very large magnetic fields to magnetize (see equation 5). Nonetheless, small magnetizations are able to change thermal conductivities and therefore ablation pressures for high $\mathrm{Z}$ plasmas $^{9}$. In the cases considered in this paper, the impact of this effect is marginal.

In contrast, at the target poles, the magnetic field remains essentially stationary, as the ablation and Nernst

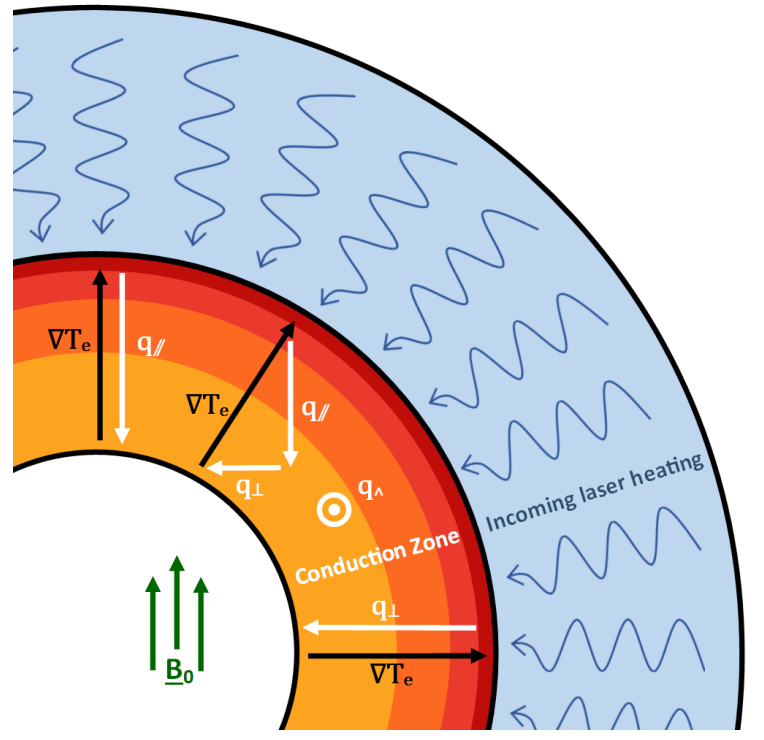

FIG. 1. Illustration showing the heat-flow components (see equation 4) in a symmetrically-driven conduction zone with an applied magnetic field up the page. The transport of magnetic field by the Nernst and cross-gradient-Nernst terms is directly analogous to the $q_{\perp}$ and $q_{\wedge}$ components (see equation 3). Capsule waist: heat-flow is perpendicular to the field lines but the magnetic field is advected away from the conduction zone, giving low magnetizations. Capsule pole: field lines remain stationary, magnetizing the conduction zone throughout the laser drive, but the heat-flow is purely along field lines and therefore remains unmodified. Once the capsule drive is perturbed from this symmetric configuration, a component of $q_{\perp}$ arises at the pole and the magnetic field changes the thermal conduction.

velocities are approximately along the field lines. The magnetic field persists at its applied strength, which can be enough to magnetize the low density and high temperature conduction zone. However, due to the field orientation, magnetization at the capsule poles only suppresses the non-radial thermal conductivities. If the capsule is driven perfectly symmetrically, the heat-flow is purely radial and the magnetic field has no impact. However, as is explored in detail in this paper, when the capsule is driven asymmetrically these lingering magnetic fields at the poles can reduce the non-radial thermal conduction.

Righi-Leduc heat-flow ( $\kappa_{\wedge}$ in equation 4$)$ is initially perpendicular to both the magnetic field and the temperature gradient, meaning that heat is transported in $\hat{\phi}$, which has no effect on azimuthally symmetric implosions. The analogous magnetic field transport, however, can twist the magnetic field. The cross-gradient-Nernst velocity is largest at the capsule waist, as the angle between the magnetic field and the temperature gradient is greatest. Moving along a single magnetic field line, the cross-gradient-Nernst transport velocity drops further away from the capsule waist. Therefore, the magnetic field line is advected in $\hat{\phi}$ more strongly at the waist than at the pole, causing a twist in between (showing up 
as a component of $\underline{B}$ in the $\underline{\hat{\phi}}$ direction). This twisting effect is similar to that simulated in laser-heated underdense plasmas ${ }^{31}$, but is yet to be seen experimentally.

This paper uses the coupled Gorgon 6,32 and Chimera ${ }^{20}$ codes. Chimera handles the 3 -D radiation hydrodynamics and laser heating, while Gorgon calculates the magnetic transport ${ }^{31}$ and anisotropic thermal conduction. All simulations in this paper use a spherical Eulerian grid. The laser drive is modeled using a ray-trace algorithm with a finite number of rays and inverse Bremsstrahlung energy deposition ${ }^{15,17}$. For simplicity, the rays are all initialized normal to the target surface. Throughout the paper, different laser heating perturbations are applied by changing the energy carried by individual rays. The initial ray positions are continuously randomized such that the imprint of a finite number of rays on the simulation is mitigated. The multi-group radiation transport of self-emitted x-rays uses $P_{1 / 3}$ automatic flux limiting, such that both diffusive and free-streaming aspects of radiation transport are captured ${ }^{5,21,29}$.

The magnetic transport includes bulk plasma advection, resistive diffusion, Nernst and cross-gradientNernst $^{31}$. The thermal conduction scheme has been specifically built to preserve heat-flow along magnetic field lines with little numerical diffusion ${ }^{27}$ and includes Righi-Leduc. A thermal and Nernst flux-limiter of 0.06 is used throughout this publication. Magnetic fields selfgenerated by the Biermann Battery term ${ }^{32}$ are found to play a secondary role to the externally-applied field and will be the subject of a future publication. The applied field dominates over the self-generated due to the Biermann field being generated perpendicular to the advection velocity; this field is advected with the bulk plasma motion and Nernst. To simplify the results in this paper, the magnetic fields generated by the Biermann Battery term are neglected.

Current-driven magnetic field advection has not been included in these calculations (although the field diffusion has been retained). The relative impact of the Nernst to Hall term can be estimated by calculating ${ }^{31}$ $\Xi \sim \gamma_{\perp}^{c} T_{e}^{\frac{5}{2}} /|\underline{B}|$. Throughout the early capsule drive $\Xi \gg 1$ in the conduction zone, indicating that neglecting the Hall term is valid; this is due to both the high temperatures and relatively low magnetic field compression. At late times, however, the magnetic flux is compressed in the cooler conduction zone regions by the Nernst effect, allowing $\Xi \approx 1$ for the case where an initial 50T field is applied. The Hall term acts in the azimuthal direction, and is expected to twist the field.

The experiment simulated throughout this paper is from a previous magnetization campaign at the OMEGA Laser Facility ${ }^{4}$. A $24 \mu m$ thick $\mathrm{CH}$ shell is filled with 10 atm $D_{2}$ gas. The laser pulse is $18 k J$ over a $1 n s$ square pulse $^{4}$. The initial outer shell radius is $430 \mu \mathrm{m}$, giving an average intensity $\approx 7 \times 10^{14} \mathrm{~W} / \mathrm{cm}^{2}$. No attempt is made in this paper to accurately model the specific perturbations present in that experiment (e.g. from laser imprint, as only 40 out of 60 OMEGA beams were used).

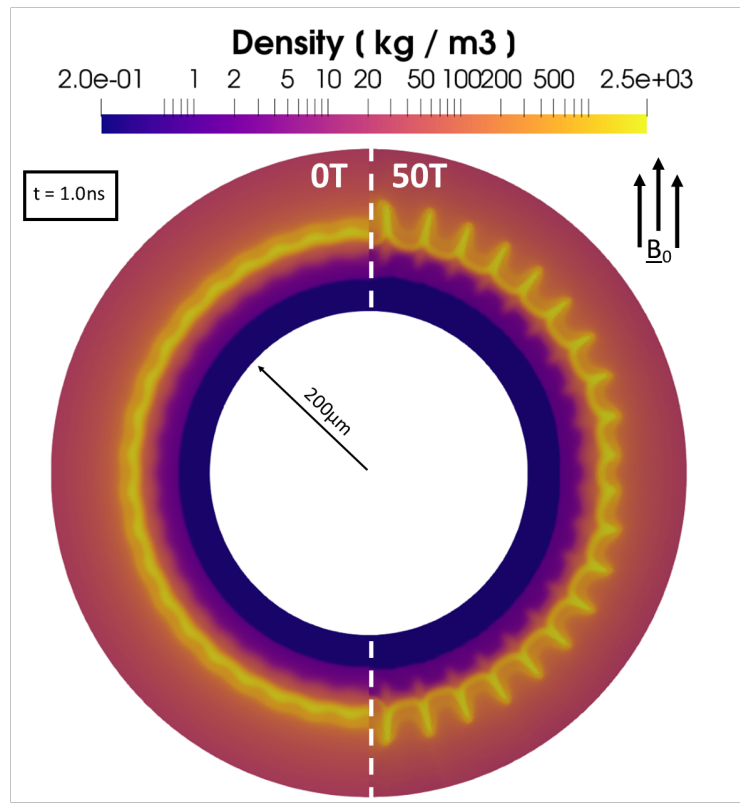

FIG. 2. Density distributions for capsules driven for $1.0 \mathrm{~ns}$ by $\mathrm{a} \pm 10 \%$ heating perturbations with mode number $k_{\theta}=40$. The left distribution is without an applied magnetic field; the right distribution is with an initial 50T magnetic field applied up the page. The perturbation growth is larger at the capsule pole in the magnetized case, as the magnetic field suppresses the non-radial thermal smoothing.

Instead, this paper uses idealized perturbations to characterize the response of direct-drive targets to an applied magnetic field. The focus is kept to the perturbation growth during the first 1.0ns, as this is the laser pulse duration. At this time the first shock has not yet converged onto the axis.

Section II uses a series of 2D single-mode simulations to study reduced thermal smoothing at the capsule poles, where magnetic field is not advected away and can increase perturbation growth rates. A strong dependence of this effect on perturbation mode number is explored. Section III then contains 3D multi-mode simulations of the capsule pole region to obtain more realistic estimates of the increase in perturbation growth rates over a wide range of wavelengths in magnetized direct-drive implosions.

\section{SINGLE-MODE PERTURBATION}

In this section a sinusoidal heating perturbation of a given mode number $k_{\theta}$ and amplitude $\pm 10 \%$ is used to drive the capsule. While the deposited energy variation at the critical density surface is $\pm 10 \%$, non-radial heat-flow in the conduction zone lowers the energy deposition perturbation at the ablation surface ${ }^{3,12}$. The smoothing of the laser perturbation through this mechanism is highly dependent on $k_{\theta}$. A $\pm 10 \%$ laser power imbalance is possible at short scales due to single- 
beam laser imprint ${ }^{22}$ and at large scales by laser beam imbalances/mispointing ${ }^{14}$. Later in this section the power imbalance is reduced to $\pm 1 \%$, with the magnetization effects persisting.

All simulations in this section use a radial cell size of $1 \mu m$ and calculate from $\theta=0, \pi$, with 360 cells in $\theta$. Reflective boundary conditions are used at the poles.

Figure 2 shows the density distribution of the target at the end of the laser drive (1.0ns) for $k_{\theta}=40$ with and without a $50 T$ applied magnetic field. For the unmagnetized case, the perturbation growth is independent of $\theta$. While the perturbation at the capsule waist is similar between the cases, the perturbation growth at the poles is enhanced by a magnetic field. The field at the pole is not ablated out of the conduction zone, allowing the nonradial heat-flow to become magnetized and suppressed.

While the magnetic field at the pole cannot be advected radially, the Nernst term still plays an important role in its non-radial transport. The magnetic flux is moved with the non-radial heat-flow, from the hot regions to the cold, where the field is compressed up to 400T. The magnetization increases from $\omega_{e} \tau_{e}=1$ at the tip of the cold dense spikes to $\omega_{e} \tau_{e}=8$ at the critical density surface. These magnetizations correspond to thermal conductivity reductions of $\kappa_{\perp} / \kappa_{\|}=0.09$ and $\kappa_{\perp} / \kappa_{\|}=0.004$ in $\mathrm{CH}$.

The density perturbations in figure 2 can be quantified by taking the radially-integrated density for both the magnetized and unmagnetized cases. The peak-to-valley difference is a factor of 5.9 greater for the $50 T$ case at the pole once the laser drive has finished.

Asymmetries in the shell density are enhanced when the target stagnates due to the deceleration-phase Rayleigh-Taylor instability. The low density bubble regions degrade performance through a lack of confinement, while the higher densities cause cold spikes to perforate the burning fuel and act as a heat sink. The perturbed drive also induces non-radial kinetic energy, which grows as the capsule converges; this component of the kinetic energy can remain unconverted into thermal energy during the stagnation, lowering the compression and increasing shape asymmetries. For the cases shown in figure 2, the peak non-radial velocities at $1.0 \mathrm{~ns}$ are $1.4 \times 10^{4} \mathrm{~m} / \mathrm{s}$ for the unmagnetized case, increasing up to $8.1 \times 10^{4} \mathrm{~m} / \mathrm{s}$ in the $50 T$ case. These magnitudes can be compared to the peak implosion velocity at this time, which is $\approx 2.7 \times 10^{5} \mathrm{~m} / \mathrm{s}$.

Figure 3 (bottom) shows the Fourier spectrum of the radially-integrated density for magnetized and unmagnetized simulations with $\pm 1 \%$ and $\pm 10 \% k_{\theta}=20$ laser heating perturbations at $1.0 \mathrm{~ns}$. Strong peaks are present at the driving mode number, with magnetic fields enhancing the growth. Non-linear instability growth is evident in the $50 \mathrm{~T} \pm 10 \%$ spectrum through peaks at the harmonics of the applied mode. The Righi-Leduc heatflow is found to enhance the strength of the harmonics in the magnetized case, with the cold spikes deflected slightly away from radial.
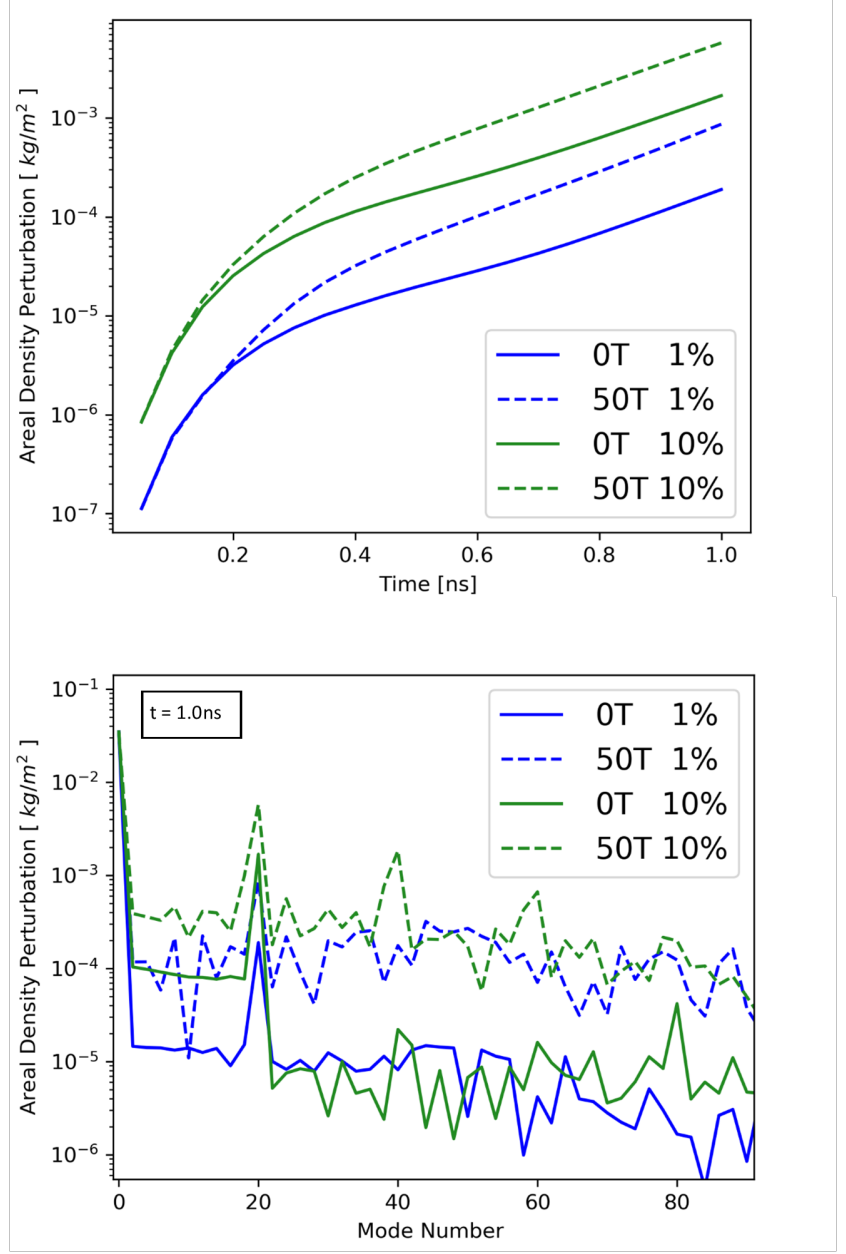

FIG. 3. Top: areal density perturbation against time for $\pm 1 \%$ and $\pm 10 \% k_{\theta}=20$ laser heating perturbations with and without 50T applied magnetic fields. The areal density perturbation is calculated using the $k=20$ peak of the Fourier transform (bottom) of the radially-integrated density for each timestep (shown here at 1.0ns). Non-linear perturbation growth is evident in Fourier spectrum peaks at multiples of the driven mode.

There is approximately an order of magnitude more noise in the magnetized areal density spectrum. A finite number of rays are used in these simulations, creating noise in the laser heating perturbation. The ray positions are randomized at each hydrodynamic timestep to lower the effect. However, the magnetized simulations enhance the noise in the same process as the single-modes are enhanced. This is evident from the fact that the $k_{\theta}>40$ areal density perturbation is similar for both the $\pm 1 \%$ and $\pm 10 \%$ simulations (except at $k_{\theta}=60$ ).

The Fourier spectrum amplitude at the driven mode number can be used as a measure of the perturbation size at a given time. This acts as an average of the perturbation size around $\theta$. Figure 3 (top) shows this measure of areal density perturbation against time for the un- 


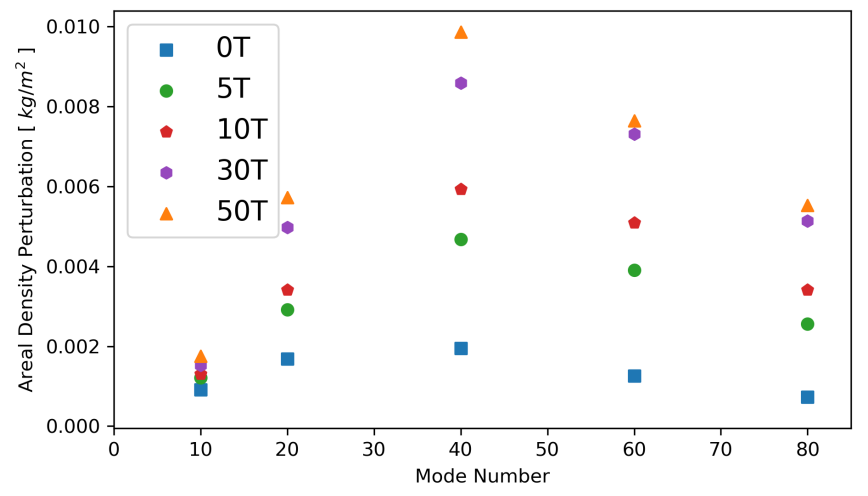

FIG. 4. Areal density at $1.0 \mathrm{~ns}$ for individual 2D simulations with different applied mode numbers and magnetic field strengths. All simulations here use $\pm 10 \%$ laser heating perturbations and can be compared with figure 5 , which uses $\pm 1 \%$. The perturbation size is calculated from the Fourier spectrum of the radially-integrated density distributions at 1.0ns. Even a small (5T) applied field increases the instability growth. This is particularly true at high mode numbers, where the perturbation evolution is governed by non-radial thermal conduction.

magnetized and magnetized simulations with $\pm 1 \%$ and $\pm 10 \% k_{\theta}=20$ laser heating perturbations. Early in the drive $(<0.1 n s)$ the magnetic fields have little impact on the perturbation size. By the end of the drive ( $\mathrm{t}=1.0 \mathrm{~ns}$ ) it is clear that the applied field has reduced the nonradial thermal conduction in the corona such that the laser drive perturbation is enhanced.

Now the relationship between perturbation growth rate, mode number $k_{\theta}$ and initial applied field strength is sought. The areal density perturbation size is parameterized using the Fourier spectrum of the radially-integrated density for each simulation. Figure 4 shows a range of 2D simulations using different applied field strengths and mode numbers, but keeping the laser heating perturbation at $\pm 10 \%$. For the unmagnetized cases, there is a peak perturbation growth at $k_{\theta}=40$. The decrease in perturbation growth for higher mode numbers is as anticipated for the ablative Rayleigh-Taylor instability ${ }^{3}$, with thermal conduction becoming more important in damping the growth. Magnetizing the capsules affects all mode numbers simulated, but the greatest amplification is for high mode numbers, which are thermal conduction dominated. It is expected from figure 4 that the shortest unstable wavelength will decrease with magnetization; this is again consistent with theory, which expects the cutoff to be set by thermal conduction effects ${ }^{2}$. Figure 4 also makes it clear that even small applied magnetic field strengths (5T) increase the perturbation growth.

The impact of magnetization is even stronger for smaller applied perturbation sizes. Figure 5 is the equivalent of figure 4 using a $\pm 1 \%$ heating perturbation. The magnetic fields enhance the growth of modes $k_{\theta}>40$ by an order of magnitude, shifting the mode number of

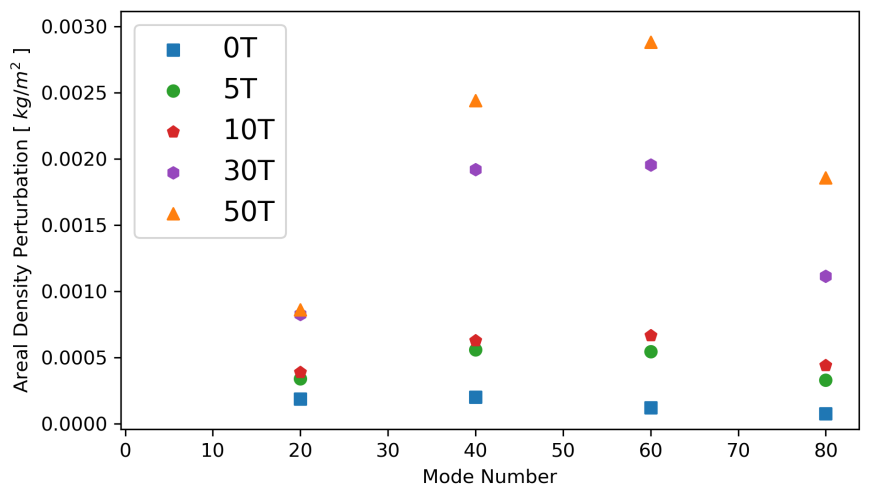

FIG. 5. Areal density perturbation size against mode number for different applied magnetic field strengths for $\pm 1 \%$ laser heating perturbations. Compared with figure 4, which uses a factor of 10 larger heating perturbation, the effect of magnetization is greater. The mode number of peak growth also increases with magnetization, from $k_{\theta}=40$ without a magnetic field to $k_{\theta}=60$ when highly-magnetized.

peak growth from $k_{\theta}=40$ to $k_{\theta}=60$. For the larger applied laser perturbation, there is naturally more nonradial heat-flow. From equation 4 it is clear that this also means more advection of magnetic fields by Nernst into the cold laser spots, thus lowering the magnetization of the conduction zone. For lower heating perturbations, the magnetic field remains in the hot regions of the conduction zone for longer and affects the non-radial thermal smoothing more strongly. In this way, it is expected that a time-dependent laser heating perturbation will see a stronger magnetization effect, as the fields will not remain stationary in the cold regions of the conduction zone, where they have a lower impact on the thermal smoothing.

\section{3D MULTI-MODE PERTURBATION}

The 2D simulations in the previous section demonstrate the effect of magnetization on isolated mode numbers and how this varies with $\theta$. However, ICF capsules are inherently three-dimensional with a multitude of mode numbers present simultaneously ${ }^{16}$. In this section, both of these changes are made by simulating a $3 \mathrm{D}$ wedge at the capsule pole. An extent of $\theta=-\pi / 6, \pi / 6$ $; \phi=-\pi / 6, \pi / 6$ is used, with 160 cells in each direction. In $3 \mathrm{D}$ there is no physical boundary condition that can be used without simulating the entire capsule when a magnetic field is applied. Instead, reflective boundary conditions are used, which do not impact the results at the pole. For ease of computation, no radiation transport is used for these 3D calculations. To further ease the computational burden, the results are analyzed halfway through the laser drive (0.5ns).

500 randomly placed sinusoidal perturbations are ap- 
plied with wavelength probability distribution $P(\lambda) \sim$ $\lambda^{2}$, with the wavelengths ranging between $0,220 \mu \mathrm{m}$. This distribution of wavelengths is chosen to reflect experimental findings that long wavelengths dominate laser imprint velocity spectral density ${ }^{22}$. The total laser heating perturbation is chosen to vary between $\pm 5 \%$.

Figure 6 shows the radially-integrated density against $\theta$ and $\phi$ with and without a 50T magnetic field at $0.5 \mathrm{~ns}$. The capsule pole (where the magnetic field is perpendicular to the shell) is located at $\theta=0, \phi=0$. Where the shell is driven more intensely, the plasma is pushed away, creating a low density spot. As in the 2D singlemode simulations, the magnetization of the conduction zone at the pole reduces the non-radial thermal smoothing, enhancing the peak-to-trough density perturbations. It is also possible to notice qualitatively that the short wavelength features are more prominent when the magnetic field is applied.

The bottom part of figure 6 shows the radiallyintegrated magnetic field strength for the case where an initial 50T field is applied. Similarities can be identified in the density distribution, with higher magnetic field strengths in the denser regions. While the magnetic field at the pole cannot move radially (along its own direction), the Nernst term and bulk plasma flow both move the field non-radially into the high density areas. As perturbations grow and non-radial bulk motion creates the high/low density regions, the field is compressed into the higher density. Assuming first that the magnetic field is just frozen into the bulk plasma motion, the percentage variation of the density and magnetic field at the pole would be expected to be the same. Instead here the areal density perturbation is just $6 \%$ compared with the magnetic field variation of $68 \%$. The Nernst term is responsible for the difference, advecting the field within the conduction zone from the hot regions to the cold. In the same way that higher magnetization suppresses the nonradial thermal conduction, it also reduces the non-radial Nernst term.

Figure 7 plots the 1D Fourier spectrum of the radiallyintegrated density for $0 \mathrm{~T}$ and $50 \mathrm{~T}$ at $0.5 \mathrm{~ns}$. It is clear that the magnetic field is enhancing the perturbation growth for a wide range of mode numbers.

\section{CONCLUSION}

The simulations in this paper predict enhanced instability growth for direct-drive implosions when a magnetic field is applied. The enhancement is greatest for shortwavelength laser heating perturbations at the pole of the capsule, where the magnetic field remains in the conduction zone throughout the laser drive. The effect is also stronger for lower amplitude heating perturbations, as the non-radial Nernst advection of the magnetic field is slower.

It is important to note that the experiment simulated here did record an increase in yield and ion tempera-
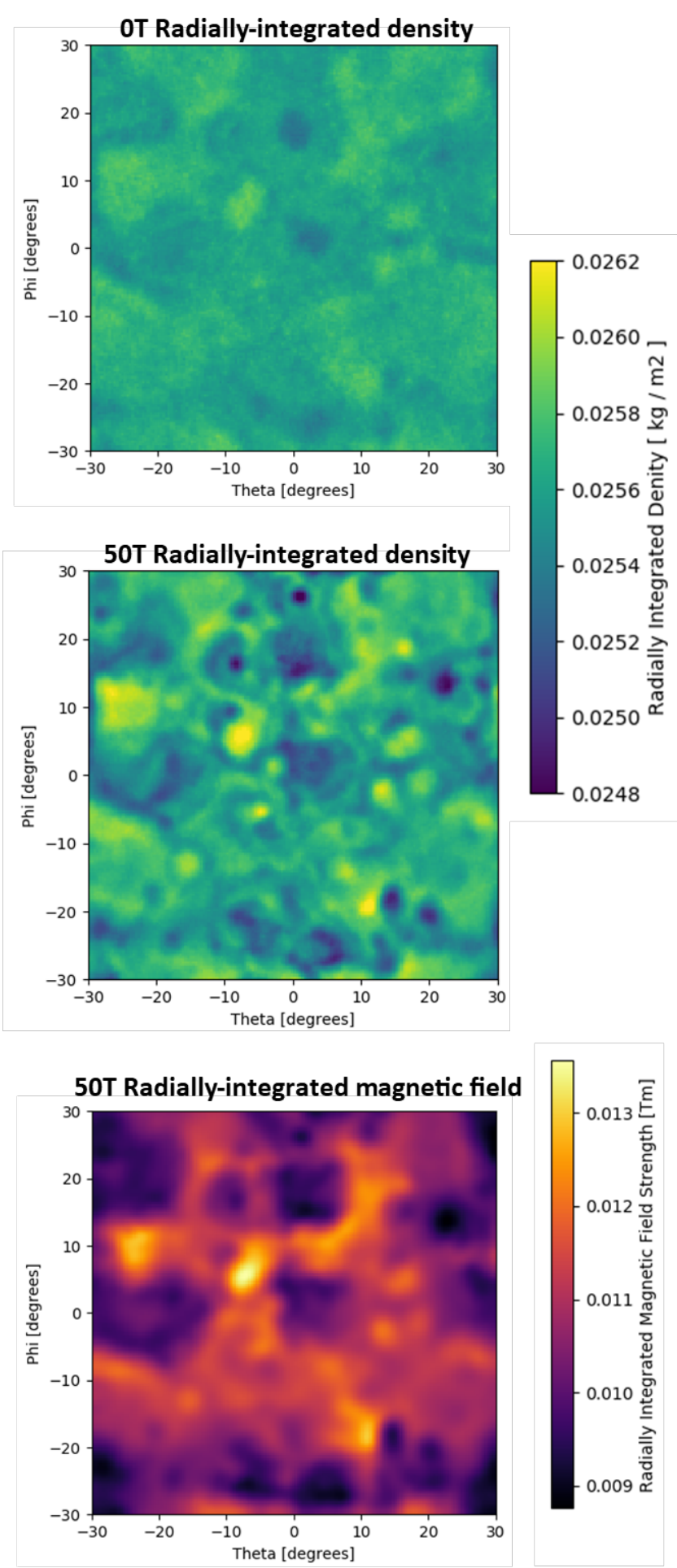

FIG. 6. Radially-integrated density at $0.5 \mathrm{~ns}$ for 3D simulations without (top) and with (middle) a 50T applied magnetic field. The capsule pole is at the image centre. The perturbation growth is enhanced when the magnetic field is applied. There is also a noticeable increase in the presence of highmode features, which is confirmed quantitatively in figure 7 . Bottom: radially-integrated magnetic field strength for an initial applied field of 50T. The field is compressed into the cold (higher radially-integrated density) regions by the Nernst effect. 


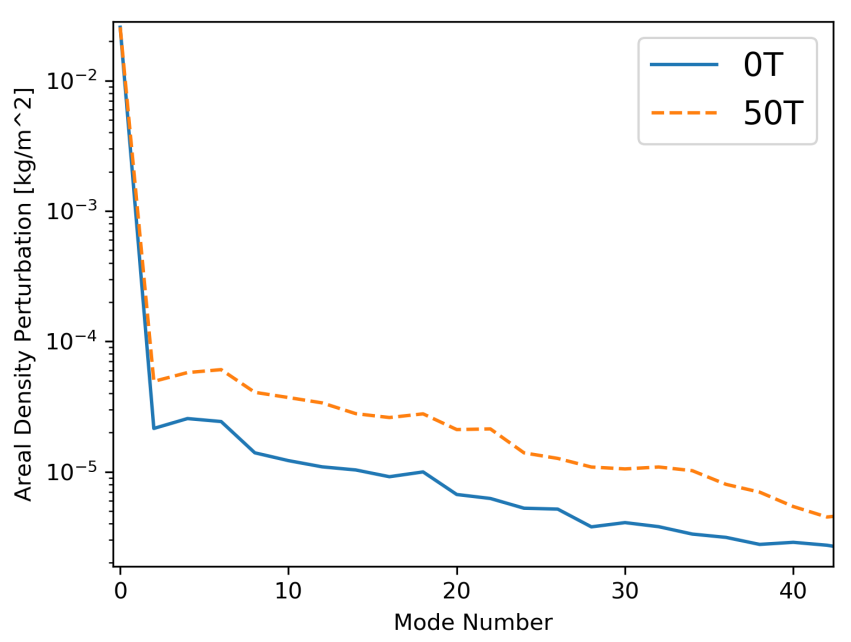

FIG. 7. 1-D line-outs through the Fourier transforms of radially-integrated density profiles from 3-D simulations using multi-mode laser heating perturbations. The applied magnetic field increases the growth of a wide range of mode numbers.

ture when magnetized ${ }^{4}$. No attempt has been made to accurately model the perturbation sources for that particular experiment, so it remains unclear whether the simulations here over-predict the perturbation enhancement or if the yields in these low convergence targets are insensitive to the short wavelength perturbation growth. Nonetheless, the enclosed results are problematic for the hope of high-yield magnetized implosions on the OMEGA Laser Facility, as the necessary increases in convergence ratio will result in a greater dependence on the target symmetry. Previous simulations have constrained the laser beam pointing uniformity for unmagnetized cryogenic implosions ${ }^{14}$ : a beam mispointing standard deviation of $10 \mu \mathrm{m}$ gives approximately a $10 \%$ reduction in yield. The results in this paper indicate that an applied magnetic field of $50 \mathrm{~T}$ will harden this constraint by an order of magnitude, requiring consistent beam pointing on the scales of $1 \mu \mathrm{m}$ for a similar yield degradation. Note that the simulations in this paper do not simulate the stagnation of the implosion, when the magnetic fields are beneficial to the implosion performance ${ }^{23,30}$.

The simulations in this paper suggest that experiments measuring the magnetized perturbation growth are essential. As the enhancements are greatest for short wavelengths, which are hard to image directly, it may be necessary to indirectly measure their effect through enhanced self-emission due to high-Z mix. In a series of experiments, dopant could be applied either at the shell pole or the waist in order to record differences in shortwavelength mixing in these regions.

It is important to note that the effects described here are not anticipated to occur for indirectly-driven implosions, as the radiation-dominated ablation regions are insensitive to electron magnetization effects. Also, the dominant perturbation enhancement effect at the pole is not relevant to cylindrical implosions, such as the miniMagLIF campaign ${ }^{8}$, as in that case the applied magnetic field is everywhere perpendicular to the target surface normal.

Kinetic effects resulting from long electron mean-freepaths have been neglected in these extended-MHD calculations. 2D Vlasov-Fokker-Plank simulations are required to ascertain the overall impact. These calculations of direct-drive perturbations are computationally tractable, as shown by Hill \& Kingham ${ }^{12}$, but were only investigated for self-generated magnetic fields. The overall impact of kinetics is unclear; while the magnetic field enhances the temperature gradient in the MHD simulations (making non-local electrons more important), the magnetization of the electrons acts to make the plasma transport more localized.

\section{ACKNOWLEDGEMENTS}

This work was performed under the auspices of the U.S. Department of Energy by Lawrence Livermore National Laboratory under Contract DE-AC52-07NA27344 and by the LLNL-LDRD program under Project Number 20-SI-002. The simulation results were obtained using the Imperial College High Performance Computer Cx1.

\section{REFERENCES}

${ }^{1}$ H. Azechi, T. Sakaiya, S. Fujioka, Y. Tamari, K. Otani, K. Shigemori, M. Nakai, H. Shiraga, N. Miyanaga, and K. Mima. Comprehensive Diagnosis of Growth Rates of the Ablative RayleighTaylor Instability. Physical Review Letters, 98(4):045002, January 2007. doi:10.1103/PhysRevLett.98.045002. URL https:// link.aps.org/doi/10.1103/PhysRevLett.98.045002. Publisher: American Physical Society.

${ }^{2}$ R. Betti, M. Umansky, V. Lobatchev, V. N. Goncharov, and R. L. McCrory. Hot-spot dynamics and deceleration-phase Rayleigh-Taylor instability of imploding inertial confinement fusion capsules. Physics of Plasmas, 8(12):5257-5267, December 2001. doi:10.1063/1.1412006. URL http://aip.scitation. org/doi/10.1063/1.1412006. Publisher: American Institute of Physics.

${ }^{3}$ Stephen E. Bodner. Critical elements of high gain laser fusion. Journal of Fusion Energy, 1(3):221-240, July 1981. ISSN 1572-9591. doi:10.1007/BF01050355. URL https://doi.org/ 10.1007/BF01050355.

${ }^{4}$ P Y Chang, G Fiksel, M Hohenberger, J P Knauer, R Betti, F J Marshall, and D D Meyerhofer. Fusion Yield Enhancement in Magnetized Laser-Driven Implosions. Physical Review Letters, 035006(July):2-5, 2011. doi:10.1103/PhysRevLett.107.035006.

${ }^{5}$ J. P. Chittenden, B. D. Appelbe, F. Manke, K. McGlinchey, and N. P. L. Niasse. Signatures of asymmetry in neutron spectra and images predicted by three-dimensional radiation hydrodynamics simulations of indirect drive implosions. In Physics of Plasmas, volume 23, pages 052708-052708, 2016. doi: 10.1063/1.4949523. URL http://scitation.aip.org/content/ aip/journal/pop/23/5/10.1063/1.4949523. Issue: 5 .

${ }^{6}$ A. Ciardi, S. V. Lebedev, A. Frank, E. G. Blackman, J. P. Chittenden, C. J. Jennings, D. J. Ampleford, S. N. Bland, S. C. 
Bott, J. Rapley, G. N. Hall, F. A. Suzuki-Vidal, A. Marocchino, T. Lery, and C. Stehle. The evolution of magnetic tower jets in the laboratory. In Physics of Plasmas, volume 14, November 2007. doi:10.1063/1.2436479. URL http://arxiv.org/abs/ astro-ph/0611441. Issue: 056501.

${ }^{7}$ J. R. Davies, R. Betti, P.-Y. Chang, and G. Fiksel. The importance of electrothermal terms in Ohm's law for magnetized spherical implosions. Physics of Plasmas, 22(112703), November 2015. doi:10.1063/1.4935286. URL http://scitation.aip.org/ content/aip/journal/pop/22/11/10.1063/1.4935286.

${ }^{8}$ J. R. Davies, D. H. Barnak, R. Betti, E. M. Campbell, P.Y. Chang, A. B. Sefkow, K. J. Peterson, D. B. Sinars, and M. R. Weis. Laser-driven magnetized liner inertial fusion. Physics of Plasmas, 24(6):062701, June 2017. ISSN 1070-664X. doi:10.1063/1.4984779. URL https://aip.scitation.org/doi/ full/10.1063/1.4984779. Publisher: American Institute of Physics.

${ }^{9}$ E. M. Epperlein and M. G. Haines. Plasma transport coefficients in a magnetic field by direct numerical solution of the Fokker-Planck equation. Physics of Fluids, 29(4), 1986. doi: 10.1063/1.865901. URL http://scitation.aip.org/content/ aip/journal/pof1/29/4/10.1063/1.865901.

${ }^{10}$ O. V. Gotchev, P. Y. Chang, J. P. Knauer, D. D. Meyerhofer, O. Polomarov, J. Frenje, C. K. Li, M. J.-E. Manuel, R. D. Petrasso, J. R. Rygg, F. H. Séguin, and R. Betti. Laser-Driven Magnetic-Flux Compression in High-Energy-Density Plasmas. Physical Review Letters, 103(21):215004, November 2009. doi: 10.1103/PhysRevLett.103.215004. URL https://link.aps.org/ doi/10.1103/PhysRevLett.103.215004. Publisher: American Physical Society.

${ }^{11}$ M G Haines. Heat flux effects in Ohm's law. In Plasma Physics and Controlled Fusion, volume 28, pages 1705-1716, 1986. doi: 10.1088/0741-3335/28/11/007. Issue: 11.

${ }^{12}$ D. W. Hill and R. J. Kingham. Enhancement of Pressure Perturbations in Ablation due to Kinetic Magnetised Transport Effects under Direct-Drive ICF relevant conditions. Arxiv, December 2017. URL http://arxiv.org/abs/1712.02663.

${ }^{13} \mathrm{M}$ Hohenberger, P. Chang, G. Fiksel, J. P. Knauer, R. Betti, F. J. Marshall, F. Seguin, C. K. Li, M. J E Manuel, and R D Petrasso. Magnetic Flux Compression Experiments on OMEGA. (March), 2011. Publisher: University of Rochester.

${ }^{14}$ S. X. Hu, V. N. Goncharov, P. B. Radha, J. A. Marozas, S. Skupsky, T. R. Boehly, T. C. Sangster, D. D. Meyerhofer, and R. L. McCrory. Two-dimensional simulations of the neutron yield in cryogenic deuterium-tritium implosions on OMEGA. Physics of Plasmas, 17(10):102706, October 2010. ISSN 1070-664X. doi: 10.1063/1.3491467. URL https://doi.org/10.1063/1.3491467. Publisher: American Institute of Physics.

${ }^{15} \mathrm{~J}$. B. Huba. NRL FORMULARY, 2013.

${ }^{16}$ I V Igumenshchev, D T Michel, R C Shah, E M Campbell, R Epstein, C J Forrest, V Yu Glebov, V N Goncharov, J P Knauer, F J Marshall, R L McCrory, S P Regan, T C Sangster, C Stoeckl, A J Schmitt, and S Obenschain. Three-dimensional hydrodynamic simulations of OMEGA implosions. Citation: Physics of Plasmas, 24, 2017. doi:10.1063/1.4979195. URL https://doi.org/10.1063/1.4979195.

${ }^{17}$ Thomas Kaiser. Laser ray tracing and power deposition on an unstructured three-dimensional grid. In Physical Review E, volume 61, pages 895-905, 2000. doi:10.1103/PhysRevE.61.895. Issue: 1 .

${ }^{18}$ J. P. Knauer, O. V. Gotchev, P. Y. Chang, D. D. Meyerhofer, O. Polomarov, R. Betti, J. a. Frenje, C. K. Li, M. J.-E. Manuel, R. D. Petrasso, J. R. Rygg, and F. H. Séguin. Compressing magnetic fields with high-energy lasers. Physics of Plasmas, 17 (056318), May 2010. doi:10.1063/1.3416557. URL http://aip. scitation.org/doi/10.1063/1.3416557.

${ }^{19}$ M. J E Manuel, M. Flaig, T. Plewa, C. K. Li, F. H. Séguin, J. A. Frenje, D. T. Casey, R. D. Petrasso, S. X. Hu, R. Betti, J. Hager, D. D. Meyerhofer, and V. Smalyuk. Collisional effects on Rayleigh-Taylor-induced magnetic fields. In Physics of Plas- mas, volume 22, 2015. doi:10.1063/1.4919392. Issue: 5 .

${ }^{20}$ Kristopher McGlinchey, Brian Appelbe, Aidan Crilly, Jon Tong, Christopher Walsh, and Jeremy Chittenden. Diagnostic Signatures of Performance Degrading Perturbations in Inertial Confinement Fusion Implosions. Physics of Plasmas, Accepted, 2018

${ }^{21}$ J. E. Morel. Diffusion-limit asymptotics of the transport equation, the $\mathrm{P} 1 / 3$ equations, and two flux-limited diffusion theories. Journal of Quantitative Spectroscopy and Radiative Transfer, 65(5):769-778, June 2000. ISSN 00224073. doi:10.1016/S0022-4073(99)00148-X. URL http://www. sciencedirect.com/science/article/pii/S002240739900148X.

${ }^{22}$ J. L. Peebles, S. X. Hu, W. Theobald, V. N. Goncharov, N. Whiting, P. M. Celliers, S. J. Ali, G. Duchateau, E. M. Campbell, T. R. Boehly, and S. P. Regan. Direct-drive measurements of laser-imprint-induced shock velocity nonuniformities. Physical Review E, 99(6):063208, June 2019. doi: 10.1103/PhysRevE.99.063208. URL https://link.aps.org/ doi/10.1103/PhysRevE.99.063208. Publisher: American Physical Society.

${ }^{23}$ L. J. Perkins, D. D.-M Ho, B. G. Logan, G. B. Zimmerman, M. A. Rhodes, D. J. Strozzi, D. T. Blackfield, and S. A. Hawkins. The potential of imposed magnetic fields for enhancing ignition probability and fusion energy yield in indirect-drive inertial confinement fusion. Physics of Plasmas, 24(6):062708-062708, June 2017. doi:10.1063/1.4985150. URL http://aip.scitation.org/ doi/10.1063/1.4985150. Publisher: AIP Publishing LLC.

${ }^{24}$ P. B. Radha, V. N. Goncharov, T. J. B. Collins, J. A. Delettrez, Y. Elbaz, V. Yu. Glebov, R. L. Keck, D. E. Keller, J. P. Knauer, J. A. Marozas, F. J. Marshall, P. W. McKenty, D. D. Meyerhofer, S. P. Regan, T. C. Sangster, D. Shvarts, S. Skupsky, Y. Srebro, R. P. J. Town, and C. Stoeckl. Two-dimensional simulations of plastic-shell, direct-drive implosions on OMEGA. Physics of Plasmas, 12(3):032702, February 2005. ISSN 1070-664X. doi: 10.1063/1.1857530. URL https://aip.scitation.org/doi/10. 1063/1.1857530. Publisher: American Institute of Physics.

${ }^{25}$ J. Sanz. Self-consistent Analytical Model of the RayleighTaylor Instability in Inertial Confinement Fusion. Physical Review Letters, 73(20):2700-2703, November 1994. doi: 10.1103/PhysRevLett.73.2700. URL https://link.aps.org/ doi/10.1103/PhysRevLett.73.2700. Publisher: American Physical Society.

${ }^{26}$ R. V. Shapovalov, G. Brent, R. Moshier, M. Shoup, R. B. Spielman, and P. A. Gourdain. Design of 30-T pulsed magnetic field generator for magnetized high-energy-density plasma experiments. Physical Review Accelerators and Beams, 22(8):080401, August 2019. doi:10.1103/PhysRevAccelBeams.22.080401. URL https://link.aps.org/doi/10.1103/PhysRevAccelBeams. 22.080401. Publisher: American Physical Society.

${ }^{27}$ Prateek Sharma and Gregory W. Hammett. Preserving monotonicity in anisotropic diffusion. Journal of Computational Physics, 227(1), November 2007. ISSN 0021-9991. doi: 10.1016/j.jcp.2007.07.026. URL https://linkinghub.elsevier. com/retrieve/pii/S0021999107003233.

${ }^{28}$ V. A. Smalyuk, S. X. Hu, J. D. Hager, J. A. Delettrez, D. D. Meyerhofer, T. C. Sangster, and D. Shvarts. Rayleigh-Taylor Growth Measurements in the Acceleration Phase of Spherical Implosions on OMEGA. Physical Review Letters, 103(10):105001, September 2009. doi:10.1103/PhysRevLett.103.105001. URL https: //link.aps.org/doi/10.1103/PhysRevLett.103.105001. Publisher: American Physical Society.

${ }^{29}$ James M. Stone and Michael L. Norman. ZEUS-2D: A radiation magnetohydrodynamics code for astrophysical flows in two space dimensions. I - The hydrodynamic algorithms and tests. The Astrophysical Journal Supplement Series, 80:753790, June 1992. ISSN 0067-0049. doi:10.1086/191680. URL http://adsabs .harvard.edu/abs/1992ApJS. . 80. .753S.

${ }^{30} \mathrm{C}$ A Walsh, K Mcglinchey, J K Tong, B D Appelbe, A Crilly, M Zhang, and J P Chittenden. Perturbation Modifications by Pre-magnetisation in Inertial Confinement Fusion Implosions. 096(December):1-12, 2018. 
${ }^{31}$ C. A. Walsh, J. P. Chittenden, D. W. Hill, and C. Ridgers. Extended-magnetohydrodynamics in under-dense plasmas. Physics of Plasmas, 27(2):022103, February 2020. ISSN 1070-664X. doi:10.1063/1.5124144. URL https://aip.scitation.org/doi/10.1063/1.5124144. Publisher: American Institute of Physics.
${ }^{32}$ C.A.Walsh, J.P.Chittenden, K. McGlinchey, N.P.L.Niasse, and B.D.Appelbe. Self-Generated Magnetic Fields in the Stagnation Phase of Indirect-Drive Implosions on the National Ignition Facility. Physical Review Letters, 118(15):155001-155001, April 2017. doi:10.1103/PhysRevLett.118.155001. URL http: //link.aps.org/doi/10.1103/PhysRevLett.118.155001. Publisher: American Physical Society. 